\title{
Estimating the second-stage sample size and the most probable number of hot spots from a first-stage sample of heavy-metal contaminated soil
}

\author{
Chuhsing Kate Hsiao ${ }^{a, *}$, Kai-Wei Juang ${ }^{\text {b,c }}$, Dar-Yuan Lee ${ }^{\mathrm{c}}$ \\ ${ }^{a}$ Division of Biostatistics, Graduate Institute of Epidemiology, National Taiwan University, No. 1, \\ Jen-Ai Rd., Section 1, Room 1542, Taipei 10018, Taiwan \\ ${ }^{\mathrm{b}}$ Energy and Resources Laboratories, Industrial Technology Research Institute, Chutung, \\ Hsinchu, Taiwan \\ ${ }^{\mathrm{c}}$ Graduate Institute of Agricultural Chemistry, National Taiwan University, Taipei, Taiwan
}

Received 15 April 1999; received in revised form 12 August 1999; accepted 29 September 1999

\begin{abstract}
This article focuses on estimation of the second-stage sample size after collecting a first set of observations in contaminated soils. There are situations where investigators are not satisfied with the results from the first-stage sampling. For instance, it is possible that the number of hot spots of pollutants identified so far does not reflect properly what the investigators expected. In that case, further sampling may be required. We propose the use of Bayes' theorem and conditional probabilities to estimate the necessary sample size. One main advantage of this approach is the ability to use information from the previous investigation as well as expert opinions. The distribution of size of the second sample is formulated based on both the size of the first-stage sampling and confidence in the number of hot spots among the first-stage observations. The estimate of the required sample size is further derived from that distribution. A real data set of the heavy-metal contaminated soil located in Taoyuan County, Taiwan was used for illustration. When confidence in the first-stage sampling does not exceed 0.5 , the chance of detecting more hot spots is 0.82 at least. In a simulation study, the probability of successfully identifying more hot spots in the second-stage sampling can be as high as 0.7 under reasonable prior assumptions. These indicate that our approach is of potential interest for estimating the second-stage sample
\end{abstract}

\footnotetext{
${ }^{*}$ Corresponding author. Tel.: +011-886-2-23970800, ext. 8372; Fax: +011-886-2-23418562.

E-mail address: ckhsiao@ha.mc.ntu.edu.tw (C.K. Hsiao).
} 
size and obtaining more hot spots in contaminated soils. (C) 2000 Elsevier Science B.V. All rights reserved.

Keywords: Bayesian approach; hot spot; sampling strategy; soil sampling; two-stage sampling

\section{Introduction}

When a remedial cleanup at a potentially contaminated site is of interest, it is always a question whether any highly contaminated spots, so-called hot spots or spatial outliers (Flatman et al., 1988), are missing from the current sampling results. The researchers may wonder if they have found all the hot spots for remediation in the field under study. In this paper, we concentrate on the estimation of the size of the second sample, when there is not much confidence in the results from the first-stage sampling and the collection of a further sample is requested.

Hot spots may have greater influences on the assessment of environmental hazards than the average contaminant concentrations (Christakos and Killam, 1993; Juang and Lee, 1998). For instance, hot spots may be a major cause of contaminated groundwater (Turnberg, 1996). To reduce the contamination and to control the cost of doing so, these spots should be the prime candidates for remediation. However, the spatial distribution of heavy metals in contaminated soil usually show heterogeneity (Juang and Lee, 1999a). It is therefore difficult to describe spatial correlation among hot spots. Similarly, optimization of a sampling scheme using geostatistics (van Groenigen and Stein, 1998) may not be suitable for finding hot spots. In addition, if there is no information about the number or location of pollution sources, one could not possibly obtain the hot spots under limited costs or survey. In this situation, a conditional random sampling paradigm can be adopted to search for more hot spots. In practice, several observations may be taken first and the spots with higher contaminant concentrations are then identified for further investigation. It is possible, however, that investigators are neither confident nor satisfied with the results. Even systematic grid sampling could have overlooked the immobile hot spots between grid nodes. Sampling with closer spacing between grid nodes is certainly an alternative to locating all highly contaminated spots; but this may not be cost-effective (Byrnes, 1994).

We discuss in this paper that when further data are to be collected under a given set of previous observations, an estimated number of points the researchers should collect to discover more hot spots for remediation. In other words, based on the information and observations of the first-stage sampling, we need to evaluate the number of points that are to be collected as the second data set for the purpose of remediation. The sampling scheme we introduce here will follow the Bayesian paradigm. That is, the estimate introduced for the sample 
size of the second-stage will be derived based on the results from the first-stage sampling via the Bayesian approach (Edwards et al., 1963). This approach utilizes the prior knowledge regarding the parameter of interest and formulates a posterior probability distribution for statistical inference (Gelman et al., 1996; Ho and Smith, 1997). In summary, the results of the first-stage sampling and any previous knowledge are considered in the prior distribution and used to obtain the posterior distribution of the size of the second-stage sampling. Next, an estimate of the size of the second data set is derived. The researcher can continue to collect more observations. After obtaining the measurements of the second data set, more hot spots may be identified. In Section 2, we will discuss an application that motivates this research, introduce the methodology, and explain how the Bayesian approach is employed. The probability distributions of $n_{2}$, size of the sample to be collected, and $M$, total number of hot spots, will be formulated and estimates will be derived. We will also examine the sensitivity of estimates when prior information varies. In other words, to investigate the change in estimates when taking into account various uncertainties in the first-stage sampling. In Section 3, we will give a simulation study to illustrate how well this procedure performed in obtaining more hot spots. Some discussions and recommendations are given in Section 4. It will be shown that our proposed sampling plan can provide a good estimate of the sample size, and further identify more hot spots for remediation when information from the first-stage sampling is properly utilized.

\section{Application and methodology}

Based on the first $n_{1}$ observations and the number $A$ of hot spots identified among them, we begin by setting up the probability distribution function of $n_{2}$ and formulating the 'prior' distribution of the total number of hot spots, $M$. We use the word 'prior' to indicate the available knowledge prior to any information to be collected in the next stage of sampling. Based on the prior and the likelihood, we then introduce the estimate of the size of the future sample via Bayes' theorem. Notations and the corresponding meanings are listed in Table 1 for the ease of reference.

\subsection{A case study of contaminant site}

The study site was a paddy field about 5 ha in area, situated in Taoyuan County, Taiwan. The soil was contaminated by cadmium contained in the discharge of a chemical plant. Measurements of cadmium concentrations were described in detail in a study by Juang and Lee (1999b). As stated there, a short interval between sampling grid nodes may help to locate all hot spots and miss none. Following the definition of the probability of missing any hot spots by 
Table 1

Notations and the corresponding meanings

\begin{tabular}{ll}
\hline Notations & Meanings \\
\hline$N$ & Population size \\
$n_{1}$ & Size of the first sample \\
$n_{2}$ & Size of the second sample \\
$\hat{n}_{2}$ & Estimate of the second sample size \\
$M$ & Number of hot spots in $N$ \\
$A$ & Number of hot spots in $n_{1}$ \\
$a$ & Confidence in $M=A$ \\
$K$ & Maximum number of hot spots \\
\hline
\end{tabular}

Zirschky and Gilbert (1984), that probability for this study region is less than $10 \%$ if the sampling interval is set to $25 \mathrm{~m}$, which results in a total number of 78 points (Fig. 1). This probability indicates that almost no hot spot would be possibly missed under their assumptions. However, when these assumptions are not satisfied or are hard to check, calculation of this probability would be infeasible. Furthermore, when financial resources are limited, it may not be possible to dig out 78 points for the whole area. On the other hand, if we can start with a wider grid of $50 \mathrm{~m}$ spacing for example, and collect the soil sample, we need to simply sample 18 points rather than 78 points. Therefore, the question becomes how many more points would be necessary based on the results of the first-stage sampling and how many more hot spots one could find

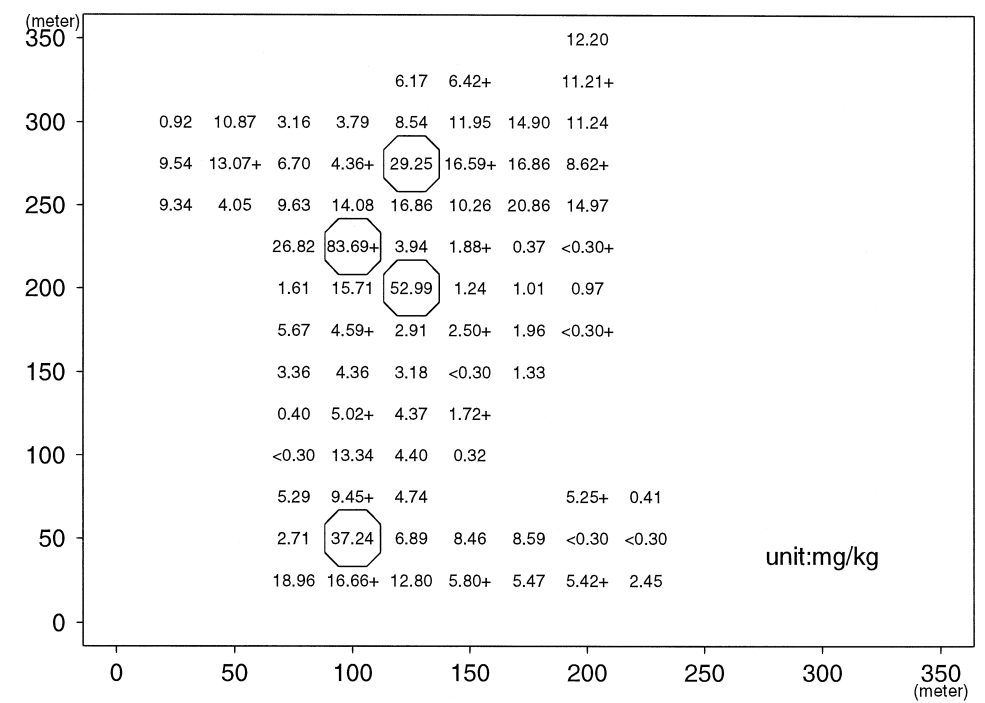

Fig. 1. The numbers are concentration levels of 78 points with four hot spots surrounded by a single octagon. The 18 values followed by a + sign are the first observations. Every two points are $25 \mathrm{~m}$ apart. 
in the study region. In other words, if the observations taken at the first stage are from wide grid spacing, one can sample further points at the second stage to find more hot spots. In the following we will describe the methodology for estimating the size of the second-stage sampling.

The study region with gridding interval spaces of $50 \mathrm{~m}$ has 18 points, while with gridding interval space of $25 \mathrm{~m}$ results in $N=78$ points in total and $M=4$ hot spots. The Cd concentrations of the 78 points are indicated in Fig. 1. The 18 values followed by a + sign are $50 \mathrm{~m}$ apart and are considered as the observations of the first-stage sample. There are some high-valued data surrounded by low-valued observations. These may be the hot spots (Juang and Lee, 1999a) of interest. Under asymptotic normality assumption, observations that are three standard errors away from the mean are considered outliers. In this study, when one observation exceeds its nearest four neighboring observations by three standard errors (s.e., where s.e. $=\mathrm{sd} / \sqrt{78}$ ) or more, it will be identified as a hot spot. In the following, we explain the proposed steps that are listed in the flowchart in Fig. 2. We first set up the likelihoods for the quantities of interest, determine the prior distribution for certain parameter, and calculate the estimate of the sample size. The proposed procedure is outlined as follows.

1. Examine the first-stage sample of $n_{1}$ points.

2. Obtain $A$, the number of hot spots among $n_{1}$ points.

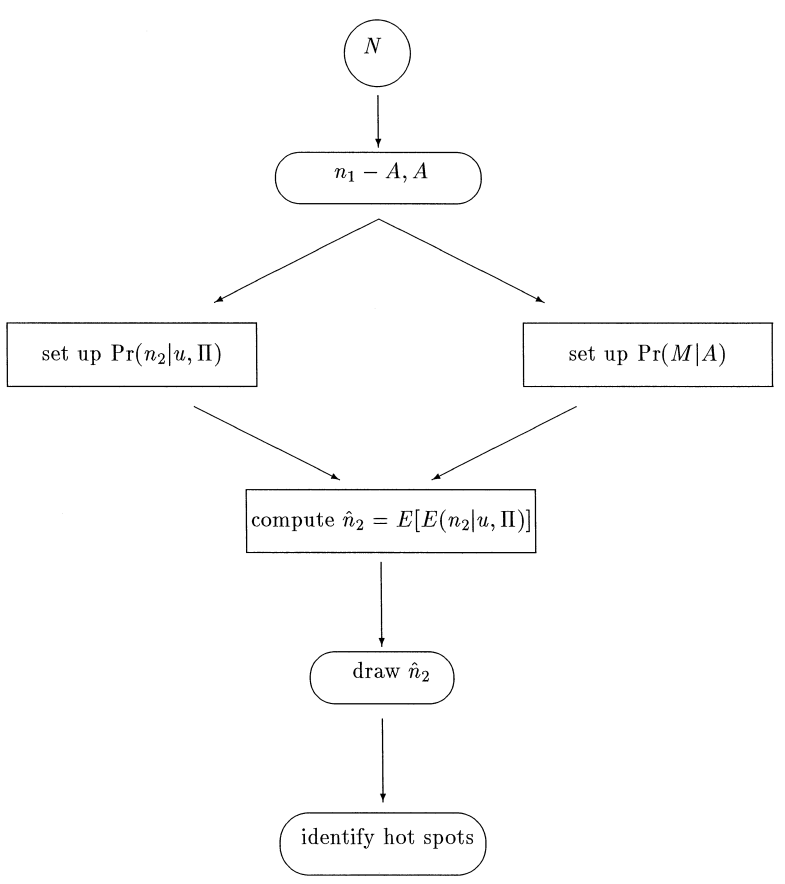

Fig. 2. The flowchart of the proposed method. 
3. Set up the probability distribution function of $n_{2}$, the size of the second sample.

4. Determine the prior distribution for $M$, the total number of hot spots, depending upon $A$.

5. Derive the posterior distribution of $n_{2}$ using information from steps 3 and 4 .

6. Compute $\hat{\mathrm{n}}_{2}$, an estimate of $n_{2}$, based on step 5 .

7. Take a random sample of $\hat{\mathrm{n}}_{2}$ points from the remaining $N-n_{1}$ locations.

8. Examine the soil contaminant levels and identify further hot spots.

\subsection{Setting up likelihood}

Let $N$ indicate the population size, i.e., the total number of available points. Let $M$ denote the true but unknown number of hot spots in the population of interest, $n_{1}$ the number of observations obtained in the first stage of sampling, $A$ the number of hot spots among the first $n_{1}$ points, and $n_{2}$ the sample size needed in the second stage to find the remaining $M-A$ hot spots. The probability density function of $n_{2}$ must depend on $A$ and the unknown $M$. Let $u$ denote the number of hot spots among the $n_{2}$ samples. Then the chance of obtaining $u$ is the same as choosing $u$ black balls from an urn of $M-A$ black balls and $N-n_{1}-(M-A)$ white balls. It is indeed a hypergeometric distribution given $M, A, N, n_{1}$, and $n_{2}$, assuming no exchangeability of correlation between locations of hot spots. That is,

$$
\operatorname{Pr}\left(u \mid n_{2}, M, A, N, n_{1}\right)=\operatorname{Pr}\left(u \mid n_{2}, \Pi\right)=\frac{\left(\begin{array}{c}
M-A \\
u
\end{array}\right)\left(\begin{array}{c}
N-n_{1}-(M-A) \\
n_{2}-u
\end{array}\right)}{\left(\begin{array}{c}
N-n_{1} \\
n_{2}
\end{array}\right)}
$$

where the notation $\left(\begin{array}{l}a \\ b\end{array}\right)$ stands for $a ! /[b !(a-b) !], \Pi$ the union of $\left(M, A, N, n_{1}\right)$, and $N$ and $n_{1}$ are constants. Though Eq. 1 is a probability measure in $u$, our focus is on the value of $n_{2}$ that is unknown. Assuming all the remaining hot spots are included in $n_{2}$, then $u=M-A$ and therefore $n_{2}$ cannot be less than $M-A$. We have, by Bayes' theorem,

$$
\operatorname{Pr}\left(n_{2} \mid u, \Pi\right)=\left[\operatorname{Pr}\left(n_{2} \mid u, M, A, N, n_{1}\right)\right]=\frac{\operatorname{Pr}\left(u \mid n_{2}, \Pi\right) \operatorname{Pr}\left(n_{2} \mid \Pi\right)}{\sum_{n_{2}=M-A}^{N-n_{1}} \operatorname{Pr}\left(u \mid n_{2}, \Pi\right) \operatorname{Pr}\left(n_{2} \mid \Pi\right)}
$$

where $\operatorname{Pr}\left(n_{2} \mid \Pi\right)=\operatorname{Pr}\left(n_{2} \mid M, A, N, n_{1}\right)$ represents the possibility of collecting $n_{2}$ observations after the first-stage sampling and is considered as the prior (prior to the second-stage sampling) distribution of $n_{2}$. Any prior information of $n_{2}$ can 
be used to formulate this prior distribution. When one has no prior preference among all the possible values of $n_{2}$, a uniform prior,

$$
\begin{aligned}
& \operatorname{Pr}\left(n_{2} \mid \Pi\right)=\left[\left(N-n_{1}\right)-(M-A)+1\right]^{-1} \\
& \text { for } n_{2}=M-A, \ldots, N-n_{1},
\end{aligned}
$$

can be considered. If one has preference among the values of $n_{2}$, a different prior can be chosen accordingly. For instance, the range of $n_{2}$ can start from any number instead of $M-A$ and therefore its distribution may vary.

Using the above uniform prior, the distribution of $n_{2}$ now becomes

$$
\begin{aligned}
\operatorname{Pr}\left(n_{2} \mid u, \Pi\right) & =\frac{n_{2} !}{\left(n_{2}-u\right) !}\left[\sum_{y=M-A}^{N-n_{1}} \frac{y !}{(y-u) !}\right]^{-1} \\
& =\frac{n_{2} !}{\left(n_{2}-u\right) !}\left[\sum_{y=0}^{N-n_{1}-u} \frac{(y+u) !}{y !}\right]^{-1}
\end{aligned}
$$

where $n_{2}$ ranges from $M-A$ to $N-n_{1}$. This probability $\operatorname{Pr}\left(n_{2} \mid u, \Pi\right)$ is indeed an inverse hypergeometric distribution (Johnson et al., 1993, p. 241). The value of $n_{2}$ starts with $M-A$ because one needs at least that many points to find the missing hot spots. For the remaining of this paper, we will use $u$ to denote $M-A$. In other words, we hope to identify all the unfound hot spots in the second-stage sampling. Obviously the distribution of $n_{2}$ depends not only on $M$ and $\left(N-n_{1}\right)$ but also on the results, e.g., $A$, from the first-stage sampling. However, once the first-stage sampling is completed, $N, n_{1}$, and $A$ become known, and the only parameter is $M$ (or $u=M-A$ ).

From Eq. 2, it can be shown that the distribution of $n_{2}$ is maximized at the point $n_{2}=N-n_{1}$. This indicates, as expected, that the probability of finding the remaining $u$ hot spots would be highest when all the remaining grids are sampled. However, it may be too costly to dig out all the nodes to find all the highly contaminated ones and we propose an alternative in the following. We use Eq. 2 as the likelihood, and combine the information about $M$ (which will be formulated next) to derive an estimate of $n_{2}$. In Eq. 2, the number $M$ is unknown and considered a random quantity. We will discuss in Section 2.3 how to introduce the uncertainty about $M$ using probability functions.

\subsection{Setting up the prior for number of hot spots}

After collecting the first $n_{1}$ observations, we have preliminary knowledge about the total number of hot spots. For instance, the number of hot spots observed among $n_{1}$ points must be greater than or equal to $A$. Based on this information, a probability distribution function $\operatorname{Pr}(M \mid A)$ for $M$, called the prior for $M$, can be constructed. The prior distribution of $M$ quantifies the information available prior to the collection of the second data set, and represents the information about the value of $M$ after the first stage of sampling is finished. Therefore, the prior should depend on the number of hot spots identified among 
$n_{1}$. It is also worth noticing that constructing a prior for $M$ is the same as constructing a prior for $u$ because $u=M-A$ where $A$ is fixed and known. After the first-stage sampling, we can assign the probability weight to various possible numbers of hot spot. The number $M$ must be bounded from above by $K$, which stands for the acceptable maximum number of hot spots in the region. Beyond that number, one may prefer to dig out the whole region than a few grid nodes for remediation. For example, if we assume the maximum number of hot spots is not greater than $1 / 10$ of the total number of points $N$, then $M$ will range from $A$ to $[N / 10]$ where $[N / 10]$ is the largest integer less than or equal to $N / 10$. Putting equal confidence in results from the first- and second-stage sampling, we formulate the prior which assigns half probability $a=0.5$ for $M=A$ and half for $M$ in $\{A+1, \ldots,[N / 10]\}$. We further assign probability $\alpha$ for $M=A+1$, and $\alpha r^{k}$ for $M=A+k+1$ where $k$ is any integer between zero and the largest integer less than $[N / 10]-A-1$. By changing the values of $a, \alpha$ and $r$, one can have different prior distributions for $M$, representing various degrees of belief in $M$ before the second-stage sampling is carried out. For instance, the more confidence one has in $M=A$, the larger $a$ will be. When one considers the contrast between the possibilities of $M=A+k$ and $M=A+$ $k^{\prime}$ to be strong, $r$ should be chosen close to zero. Similarly, the maximum number of hot spots and probability for $\operatorname{Pr}(M=A \mid A)$ can be changed in accordance with the subject matter under study. This prior is summarized as follows:

$$
\begin{aligned}
& \operatorname{Pr}(M=A \mid A)=a, \quad \operatorname{Pr}(M=A+1 \mid A)=\alpha, \\
& \operatorname{Pr}(M=A+2 \mid A)=\alpha r, \quad \ldots, \quad \operatorname{Pr}(M=[N / 10] \mid A)=\alpha r^{[N / 10]-A-1} .
\end{aligned}
$$

Letting $K=[N / 10]$, it can be derived that $\alpha=[(1-a)(1-r)] /\left[1-r^{K-A}\right]$ because all probabilities sum to $1\left(a+\alpha+\alpha r+\ldots+\alpha r^{K-A-1}=1\right)$.

As an illustration and for later use in the application and simulation, Table 2 lists the values of $\alpha$ when $a$ and $r$ are given at 0.2, 0.5, and 0.8, respectively and the maximum number of hot spots is assumed seven. The magnitude of $a$ indicates the strength of confidence in $A$ among the first sample. It can be seen that the value of $a$ determines the shape of the distribution and $r$ represents the different degrees of belief in consecutive numbers greater than $A$. Furthermore, when $a=0.2$ the prior has a peak at $M=(A+1)$ at whatever value $r$ takes,

Table 2

Values of $\alpha$ for given $a$ and $r$ in the prior distribution of $M$ when the maximum number of hot spots is seven

\begin{tabular}{llll}
\hline & $a=0.2$ & $a=0.5$ & $a=0.8$ \\
\hline$r=0.2$ & 0.64 & 0.40 & 0.16 \\
$r=0.5$ & 0.41 & 0.26 & 0.10 \\
$r=0.8$ & 0.22 & 0.15 & 0.05 \\
\hline
\end{tabular}


which indicates one's strongest prior belief in some $M$ greater than $A$. When $a=0.5$ or 0.8 , the prior decreases with different rates determined by $r$. This indicates the strongest confidence in $A$ but less elsewhere.

Alternatively, one could use a uniform prior. That is, $\operatorname{Pr}(M \mid A)=1 /(\lfloor N / 10\rfloor$ $-A+1)$ for $M=A, A+1, \ldots,\lfloor N / 10]$. This indicates no particular preference in any of the numbers, even after some hot spots have been observed. This particular prior will also be considered in the following sections when examining its influence on the estimation of the size of further sample. We now use the prior of $M$ and the distribution of $n_{2}$ given $u, M, A, N$ and $n_{1}$ to estimate $n_{2}$.

\subsection{Estimation}

An estimate of $n_{2}$, the size of the second-stage sampling, should depend on both the value of $A$ and the prior distribution we have set up for $M$. The proposed estimate is the following double expectation

$$
\begin{aligned}
\hat{n}_{2} & =E\left[E\left(n_{2} \mid u, \Pi\right)\right]=E\left[\sum_{n_{2}=u}^{N-n_{1}} n_{2} \cdot \operatorname{Pr}\left(n_{2} \mid u, \Pi\right)\right] \\
& =\sum_{M=A}^{\lfloor N / 10\rfloor}\left[\sum_{n_{2}=u}^{N-n_{1}} n_{2} \cdot \operatorname{Pr}\left(n_{2} \mid u, \Pi\right)\right] \cdot \operatorname{Pr}(M \mid A)
\end{aligned}
$$

where the inside expectation evaluates the conditional expectation of $n_{2}$ based on Eq. 2 with $u=M-A$ fixed, and the outside expectation equals the weighted average of the conditional expectation over all possible values of $M$ according to $\operatorname{Pr}(M \mid A)$, one's prior confidence in $M$. This estimate incorporates the information contained in the first $n_{1}$ observations. This approach provides a way to change $\hat{n}_{2}$ when there are various opinions about $M$. As we have pointed out in Section 2.3, the prior for $M$ can also be considered as a prior for $u$. In other words, the outer expectation may be taken with respect to different values of $u$.

Some may prefer to estimate the sample size by maximizing the likelihood function of $n_{2}$, namely Eq. 2. However, the likelihood always attains its maximum at $n_{2}=N-n_{1}$, which may be intuitive but offers no help. After the $n_{2}$ points are sampled and measurements for level of concentration are collected, more hot spots among these points can be identified.

\subsection{Prior selection and sensitivity analysis}

In this section, we will examine the influence of the confidence in $n_{1}$ on the estimate $\hat{n}_{2}$. Following the same notation used in Section 2.3, a denotes the confidence in the initial finding $M=A, \alpha$ denotes the confidence in $M=A+1$, $\alpha r$ denotes the confidence in $A+2, \ldots$, etc. Once we have decided $a$ and $r$, the $\alpha$ can be computed because the probabilities must sum to unity. We have 
deliberately chosen $a=0.2,0.5,0.8$ to represent different degrees of belief in $M=A$ and $r=0.2,0.5,0.8$ for various levels of confidence between consecutive numbers. Another prior we will consider is the uniform distribution, indicating equal probability weights on all possible values of $M$. Based on Eq. 3 , the corresponding estimate $\hat{n}_{2}$ is listed in Table 3 .

When $A=0$, indicating no hot spot has been found among the $n_{1}$ observations, one may put zero probability $(a=0)$ for $M=0$ if hot spots are believed to exist. In that case, the size of the second-stage sampling would be large, as shown in the first column of Table 3 . However, it decreases if the initial size $n_{1}$ is large. Our second observation is that the magnitude of $\hat{n}_{2}$ does not vary much for various $r$ when $A$ and $a$ are fixed. This is seen in any three consecutive numbers in the same column in Table 3 . It implies that only the size $n_{1}$ of the first sample and the confidence $a$ in the number of hot spots among $n_{1}$ are influential to $\hat{n}_{2}$. When $n_{1}$ is large, $\hat{n}_{2}$ is usually small, this is reasonable and intuitive. Finally, as we look through the rows as $a$ increases from 0.2 to 0.8 for fixed $A$ and $r$, the estimates decrease. This indicates that when one is quite confident (large $a$ ) in the first finding $A$, there is less need to get additional data.

We conclude from the table that $a$ has a stronger influence than $r$ in the value of $\hat{n}_{2}$. Therefore, we suggest choosing $a$ first, according to the strength of confidence one has in $A$, to decide on the prior distribution of $M$, and then calculate the size of the second sample. Alternatively, when one believes equally

Table 3

Estimates of the number of second samples $\left(\hat{n}_{2}\right)$ with respect to different priors and different size $\left(n_{1}\right)$ of initial samples. The size of population is $N=78$

\begin{tabular}{|c|c|c|c|c|c|c|c|c|c|c|c|}
\hline & \multirow{2}{*}{$\begin{array}{l}A=0 \\
a=0\end{array}$} & \multicolumn{3}{|l|}{$A=1$} & \multicolumn{3}{|l|}{$A=2$} & \multicolumn{3}{|l|}{$A=3$} \\
\hline & & & $\overline{a=0.2}$ & $a=0.5$ & $a=0.8$ & $\overline{a=0.2}$ & $a=0.5$ & $a=0.8$ & $\overline{a=0.2}$ & $a=0.5$ & $a=0.8$ \\
\hline \multirow[t]{4}{*}{$n_{1}=10$} & $r=0.2$ & 47 & 38 & 24 & 9 & 38 & 24 & 9 & 38 & 24 & 9 \\
\hline & $r=0.5$ & 50 & 40 & 25 & 10 & 40 & 25 & 10 & 39 & 25 & 10 \\
\hline & $r=0.8$ & 53 & 42 & 27 & 11 & 42 & 26 & 10 & 41 & 26 & 10 \\
\hline & uniform & 56 & 47 & 47 & 47 & 38 & 38 & 38 & 30 & 30 & 30 \\
\hline \multirow[t]{4}{*}{$n_{1}=20$} & $r=0.2$ & 40 & 32 & 20 & 8 & 32 & 20 & 8 & 32 & 20 & 8 \\
\hline & $r=0.5$ & 43 & 34 & 21 & 9 & 34 & 21 & 9 & 34 & 21 & 8 \\
\hline & $r=0.8$ & 46 & 36 & 23 & 9 & 36 & 22 & 9 & 35 & 22 & 9 \\
\hline & uniform & 48 & 40 & 40 & 40 & 33 & 33 & 33 & 26 & 26 & 26 \\
\hline \multirow{4}{*}{$n_{1}=40$} & $r=0.2$ & 26 & 21 & 13 & 5 & 21 & 13 & 5 & 21 & 13 & 5 \\
\hline & $r=0.5$ & 28 & 22 & 14 & 6 & 22 & 14 & 6 & 22 & 14 & 6 \\
\hline & $r=0.8$ & 30 & 24 & 15 & 6 & 24 & 15 & 6 & 23 & 14 & 6 \\
\hline & uniform & 31 & 27 & 27 & 27 & 22 & 22 & 22 & 17 & 17 & 17 \\
\hline \multirow[t]{4}{*}{$n_{1}=60$} & $r=0.2$ & 13 & 10 & 6 & 3 & 10 & 6 & 3 & 10 & 6 & 3 \\
\hline & $r=0.5$ & 14 & 11 & 7 & 3 & 11 & 7 & 3 & 11 & 7 & 3 \\
\hline & $r=0.8$ & 15 & 12 & 7 & 3 & 11 & 7 & 3 & 11 & 7 & 3 \\
\hline & uniform & 15 & 13 & 13 & 13 & 10 & 10 & 10 & 8 & 8 & 8 \\
\hline
\end{tabular}


about the possible values of $M$, the uniform prior can be considered, though this case may be uncommon in practice. The estimates are also listed in Table 3.

\subsection{A case study (revisited)}

We will now consider the case discussed in Section 2.1. Recall that only 18 points at $50 \mathrm{~m}$ apart were observed at the first stage and one of them was a hot spot (see also Fig. 1). In other words, $A=1$. The likelihood of $n_{2}$ can then be derived based on Eq. 2 with $M$ being the unknown parameter. Note that $u$ is also unknown because $u=M-A=M-1$ in this case. Next, we pick four prior distributions for $M$ : uniform from $A=1$ to 7 ; and $(a, r)=(0.2,0.5),(0.5,0.5)$, $(0.8,0.5)$, respectively. The uniform prior indicates no preference among all possible values for $M$, and the other three priors indicate weaker confidence in $M=A$. The $r$ in these three priors is fixed because its influence is less important than $a$, as discussed in Section 2.5. Following Eq. 3, the estimated numbers $\hat{n}_{2}$ are $41,35,22$, and 9, for the four priors respectively. The next step is then to select $\hat{n}_{2}$ points and check the contamination levels. Further hot spots will then be identified among the second sample. We run a simulation of 1000 replications under each estimate of $n_{2}$ and examine the probability of identifying more than one hot spot. These probabilities are $0.94,0.91,0.82$, and 0.42 , respectively. Taking the more rigorous standard of identifying three or four hot spots, the resulting probabilities are $0.72,0.56,0.34$, and 0.03 , respectively. The first three numbers are not small indicating a successful operational plan. The last probability 0.03 is small because it corresponds to a large $a=0.8$ and a small $\hat{n}_{2}=9$.

\section{Simulation study}

The purpose of this simulation is to illustrate the performance of the proposed method in the situation similar to the application we discussed above. It is carried out to investigate how efficient the sampling procedure is in finding more hot spots. Various situations (different $n_{1}$ and $A$ ) for the initial sample are considered and followed by sampling $\hat{n}_{2}$ points. The value of $n_{1}$ has been set to $10,20,40$, and 60 , respectively and four types of priors on $M$ are considered. The first prior assigns 0.1 probability on $M=A$ and the second is a uniform distribution with probabilities $1 / 7$ for each $M=1,2, \ldots, 7$. The third prior puts $a=0.2$ probability mass (indicating weak confidence) on $A$, and the last one assigns 0.5 probability on $A$, indicating strong confidence in the first finding. The maximum number of hot spots is $[78 / 10]=7$ by our definition, although the true value is only 4 . The $r$ is chosen as 0.5 because it has less importance on $\hat{\mathrm{n}}_{2}$ than $a$ does, as seen in Section 2. 
There are 1000 replications. For each replication, $A$ is fixed first (at $0,1,2$, or 3) and $n_{1}$ observations are chosen with the presence of exact $A$ hot spots. Based on $A$ and $n_{1}$, a sample of size $\hat{n}_{2}$ (its value can be computed based on Eq. 3 or obtained from Table 3 ) is chosen randomly among the remaining $\left(78-n_{1}\right)$ points and hot spots are identified.

We consider identifying at least three hot spots among four successful runs and compute the probabilities $\operatorname{Pr}(M \geq 3)$ of getting three or four hot spots under different settings. The probability of successfully identifying more hot spots is fairly high, as listed in Table 4. For the first three priors, the probability of finding more hot spots is high regardless of the values of $n_{1}$ and $A$, indicating good chances of success. However, when the confidence $a$ in $A$ is as strong as 0.5 , this probability can be small as indicated in the last column of Table 4, because the final result is dominated by strong confidence in $A$. Based on the results, it can be seen that the choice of prior on $M$ is important in obtaining the value of $\hat{\mathrm{n}}_{2}$ for the second sample. If one is very confident in the first finding $A$, there is not much need to get a further sample and therefore a small $\hat{n}_{2}$ and a small probability of $(M \geq 3)$ are expected. When $A=0$, there is no need to specify $a$ and thus, only uniform prior is considered and 'na' was put in the corresponding positions in Table 4 . The variance of the probability $\operatorname{Pr}(\hat{M} \geq 3)$ can be obtained by computing $p(1-p) / 1000$ where $p$ is $\operatorname{Pr}(\hat{M} \geq 3)$ in the table and 1000 is the total number of replications. For instance, when $p=0.77$, the standard deviation is only 0.01 .

Table 4

Probability of obtaining three or four hot spots $[\operatorname{Pr}(\hat{\mathrm{M}} \geq 3)]$ under four prior distributions with $r=0.5$ for all cases: (1) $a=0.1$ on $M=A$; (2) uniform on $M$; (3) $a=0.2$ on $M=A$; and (4) strong information, $a=0.5$ on $M=A$

\begin{tabular}{llllll}
\hline & & $a=0.1$ & Uniform & $a=0.2$ & $a=0.5$ \\
\hline$n_{1}=10$ & $A=1$ & 0.77 & 0.80 & 0.63 & 0.24 \\
& $A=2$ & 0.91 & 0.91 & 0.80 & 0.63 \\
& $A=3$ & 1.0 & 1.0 & 1.0 & 1.0 \\
$n_{1}=20$ & $A=0$ & na & 0.83 & na & na \\
& $A=1$ & 0.69 & 0.74 & 0.57 & 0.34 \\
& $A=2$ & 0.85 & 0.84 & 0.82 & 0.64 \\
& $A=3$ & 1.0 & 1.0 & 1.0 & 1.0 \\
$n_{1}=40$ & $A=0$ & na & 0.82 & na & na \\
& $A=1$ & 0.64 & 0.70 & 0.56 & 0.35 \\
& $A=2$ & 0.83 & 0.75 & 0.77 & 0.62 \\
& $A=3$ & 1.0 & 1.0 & 1.0 & 1.0 \\
$n_{1}=60$ & $A=0$ & na & 0.72 & na & na \\
& $A=1$ & 0.62 & 0.77 & 0.56 & 0.30 \\
& $A=2$ & 0.84 & 0.72 & 0.76 & 0.53 \\
& $A=3$ & 1.0 & 1.0 & 1.0 & 1.0 \\
& $A=0$ & na & 0.84 & na & na \\
\hline
\end{tabular}




\section{Discussions}

This paper focuses on estimating the size of the second-stage sampling to find more hot spots for remediation. Our purpose is not to locate all hot spots. In that case, one may as well dig out the whole area under study. On the contrary, we try to find as many hot spots as possible under limited labor and cost. Our procedure would be useful when budget and efficiency are of concern and when a preliminary data collection has been carried out. Our procedure utilizes the information obtained in the first set of observations, including the size $n_{1}$, the number of hot spots $A$, and the confidence in $M=A$. With this information, one can construct both the likelihood function of $n_{2}$ and the prior distribution of $M$ before proceeding with the second stage of sampling. Using the Bayesian approach, an estimate of $n_{2}$ is computed and, when the second data set is collected, more hot spots are identified. The application and the simulation described in the previous sections show that the probability of success can be high.

Our strategy is to make the best use of the first sample when a second sample is desired. In particular, all the probability distributions should be properly conditioned on the information collected among the $n_{1}$ observations. This objective can be easily achieved by Bayes' theorem. The reasoning for using the conditional probability also applies in deriving Eq. 2, the inverse hypergeometric distribution of $n_{2}$. When constructing the prior distribution of $M$, it was argued in Section 2 that the confidence $a$ in $A$ is influential. Consequently, it affects the final search of the hot spots. Its influence can be seen in Tables 3 and 4. Once one can quantify the confidence in the results from the first finding, the remaining computation should be straightforward following Eqs. 2 and 3. We conclude that both the size $n_{1}$ and confidence $a$ are influential to $\hat{n}_{2}$.

There are other considerations in the application of this procedure. First, our method requires the magnitude of $N$ to be known in advance. The number may not be constrained by the shape of the region or any construction above the soil. This number depends on both the size of the whole area and the size of each sampling unit. In fact, it can be computed by taking the ratio of the two values; we recommend this choice. The number $N$ can also be chosen based on the budget for sampling. In addition, although Tables 3 and 4 were designed for a special case with particular numbers of $n_{1}$ and $N$, generalization to other values should follow easily. Second, the choice of the prior distribution should always be problem-dependent. The following recommendations for the choice of $a$ and $r$ in the prior distribution may be helpful:

1. $r$ is not influential and use 0.5 as a reference number,

2. use small $a$ (such as 0.1 ) or uniform prior when results from the first-stage sampling are not satisfactory, 
3. use large $a$ (such as 0.5 or 0.8 ) when the investigator is very confident in the value of $A$ or when very limited budget is left for the second-stage sampling, and

4. choose $a=0.8$ over $a=0.5$ when the investigator believes that almost all hot spots have been found in $n_{1}$.

Third, note that the maximum number of hot spots in this approach has been chosen arbitrarily to be less than $1 / 10$ of $N$. This decision takes some prior knowledge about the region under study and may need an expert's judgement. The cost for the clean-up of the whole region, spacing between grid nodes, and any information about the deposition may affect the maximum number. For our data, we felt that any number larger than seven may imply a clean-up of the whole region and thus seven seems an appropriate maximum number. However, to alter the value for other applications is absolutely possible, and all computations can be carried through in the same manner. When the prior distribution puts little probability on large values of $M$, it may not matter much what the maximum number is. On the other hand, when a prior assigns large probability on large values of $M$, the choice of the maximum number would be influential. A sensitivity analysis may be necessary to give detailed information. If the investigators are not satisfied with the results from the second-stage sampling, this procedure can also be applied to the third stage of sampling. In that case, confidence in both the first and second results should be slim. Finally, note that we have assumed $u=M-A$ in the calculation of the posterior distribution of $n_{2}$, an alternative is to free the assumption and introduce uncertainty in $u$. This will require specification of the probability measure of $u$ and may bring complexity to all computations. However, the same operational plan for deriving an estimate of the second sample size should continue in a similar manner.

In a different situation, when both sampling stages are considered and designed, several other sampling techniques may be of use. The commonly used two-stage, multistage, double sampling schemes (Tenenbein, 1972), or sequential sampling (Mukhopadhyay et al., 1992) may be helpful when more investigation can be done with the first-stage sampling, including dividing them into smaller sub-units and/or using different methods for measurements (Thompson, 1992); but these would not be applicable in our situation. Composite sampling (Dorfman, 1943; Garner et al., 1988) or refined composite sampling (Shieh, 1997) deals with samples that can be pooled but may not be helpful in identifying the contaminated spots. Some references including Domburg et al. (1997) and van Groenigen et al. (1997) discuss the multistage sampling. Other literatures have focused on the choice of grid spacing. For instance, Zirschky and Gilbert (1984) have developed a waste site sampling procedure to determine the probability of finding a hot spot of a certain size given at particular grid spacing. Their approach requires a dense sampling scheme and may be time consuming when the field of interest is large. 
In this paper, we do not intend to restrict sampling to regularly spaced grid areas, or to locate the exact positions of hot spots. Although sample points in our application are all equally spaced, the procedure can work well in an application with irregular spacing points. When deriving our likelihood and prior distribution, the $M$ hot spots are assumed independent with respect to their locations. In that case, the observations among $n_{1}$ with high contamination levels may deserve more attention and further data around those may be required to detect the exact location of the pollution source. In this situation, the assumption of correlation among grid nodes or hot spots may be present and a different approach accounting for spatial correlation should be considered.

\section{Acknowledgements}

The authors are grateful to the comments of the editor and the two referees which improved the manuscript greatly.

\section{References}

Byrnes, M.E., 1994. Field Sampling Methods for Remedial Investigations. Lewis Publishers, Boca Raton, Chapter 2.

Christakos, G., Killam, B.R., 1993. Sampling design for classifying contaminant level using annealing search algorithms. Water Resour. Res. 29, 4063-4076.

Domburg, P., de Gruijter, J.J., van Beek, P., 1997. Designing efficient soil survey schemes with a knowledge-based system using dynamic programming. Geoderma 75, 183-201.

Dorfman, R., 1943. Detection of defective members of a large population. Ann. Math. Stat. 14, 436-440.

Edwards, W., Lindman, H., Savage, L.J., 1963. Bayesian statistical inference for psychological research. Psychol. Rev. 70, 193-242.

Flatman, G.T., Englund, E.J., Yfantis, A.A., 1988. Geostatistical approaches to the design of sampling regimes. In: Keith, L.H. (Ed.), Principles of Environmental Sampling. American Chemical Society, Washington, DC, pp. 73-84.

Garner, F.C., Stapanian, M.A., Williams, L.R., 1988. Composite sampling for environmental monitoring. In: Keith, L.H. (Ed.), Principles of Environmental Sampling. American Chemical Society, Washington, DC, pp. 363-374.

Gelman, A., Carlin, J.B., Stern, H.S., Rubin, D.B., 1996. Bayesian Data Analysis. Chapman \& Hall, London.

Ho, C.H., Smith, E.I., 1997. Volcanic hazard assessment incorporating expert knowledge: Application to the Yucca Mountain region, Nevada, USA. Math. Geol. 29, 615-627.

Johnson, N.L., Kotz, S., Kemp, A.W., 1993. Univariate Discrete Distributions. Wiley, New York.

Juang, K.W., Lee, D.Y., 1998. A comparison of three kriging methods using auxiliary variables in heavy-metal contaminated soils. J. Environ. Qual. 27, 355-363.

Juang, K.W., Lee, D.Y., 1999a. Geostatistical cross-validation for the design of additional sampling regimes in heavy-metal contaminated soils. J. Chin. Inst. Environ. Eng., (in press).

Juang, K.W., Lee, D.Y., 1999b. Use of quantile of rank order transformation to improve kriging estimation of heavy metal distribution in contaminated soils. Environ. Sci. Technol., Revised and resubmitted. 
Mukhopadhyay, N., Bendel, R.B., Nikolaidis, N.P., Chattopadhyay, S., 1992. Efficient sequential sampling strategies for environmental monitoring. Water Resour. Res. 28, 2245-2256.

Shieh, G.S., 1997. Refined composite sampling for acute toxicity standard. Environmetrics 8, 121-132.

Tenenbein, A., 1972. A double sampling scheme for estimating from misclassified multinomial data with applications to sampling inspection. Technometrics 14, 187-202.

Thompson, S.K., 1992. Sampling. Wiley, New York.

Turnberg, W.L., 1996. Biohazardous Waste: Risk Assessment, Policy, and Management. Wiley, New York.

van Groenigen, J.W., Stein, A., 1998. Constrained optimization of spatial sampling using continuous simulated annealing. J. Environ. Qual. 27, 1078-1086.

van Groenigen, J.W., Stein, A., Zuurbier, R., 1997. Optimization of environmental sampling using interactive GIS. Soil Technol. 10, 83-97.

Zirschky, J., Gilbert, R.O., 1984. Detecting hot spots at hazardous-waste sites. Chem. Eng. 91, 97-100. 